\title{
A Method for Solving Legendre's Conjecture
}

\author{
Hashem Sazegar \\ Department of Mathematics, Azad University of Mashhad, Mashhad, Iran \\ E-mail: h.sazegar@gmail.com
}

Received: September 29, 2011 Accepted: October 31, 2011 Published: February 1, 2012

doi:10.5539/jmr.v4n1p121 URL: http://dx.doi.org/10.5539/jmr.v4n1p121

\begin{abstract}
Legendre's conjecture states that there is a prime number between $n^{2}$ and $(n+1)^{2}$ for every positive integer $n$. In this paper we prove that every composite number between $n^{2}$ and $(n+1)^{2}$ can be written $u^{2}-v^{2}$ or $u^{2}-v^{2}+u-v$ that $u>0$ and $v \geq 0$. Using these result as well as induction and residues $(\bmod q)$ we prove Legendre's conjecture.
\end{abstract}

Keywords: Bertrand-Chebyshev theorem, Landau's problems, Goldbach's conjecture, Twin prime, Ramanujan proof

\section{Introduction}

Bertrand's postulate state for every positive integer $n$, there is always at least one prime $p$, such that $n<p<2 n$. This was first proved by Chebyshev in 1850 which is why postulate is also called the Bertrand-chebyshev theorem. Ramanujan gave a simple proof by using the properties of gamma function, which resulted in concept of Ramunajan primes (Ramanujan, 1919, p. 181-182). In 1932 Erdos published a simple proof using the Chebyshev's function and properties of binomial coefficient (Erdos, 1932, p. 194-198). Legendre's conjecture states that there is a prime between $n^{2}$ and $(n+1)^{2}$ for every positive integer $n$, which is one of the four Landau's problems. The rest of these four basic problems are:

(i) Twin prime conjecture: there are infinitely many primes $p$ such that $p+2$ is a prime.

(ii) Goldbach's conjecture: every even integer $n>2$ can be written as the sum of two primes.

(iii) Are there infinitely many primes $p$ such that $p-1$ is a perfect square?

problems (i),(ii),(iii) are open till date.

In this paper we state one proof for Legendre's conjecture.

Theorem There is at least a prime between $n^{2}$ and $(n+1)^{2}$.

We prove it by induction that if there is at least a prime between all squares less than $(x-1)^{2}$, then there is a prime between $(x-1)^{2}$ and $x^{2}$, that $x$ is a large positive integer. Assume that this is not hold, i.e all numbers in interval $(x-1)^{2}$ and $x^{2}$ are composite and we reach to a contradiction.

To proceed to this proof, firstly we use the following Lemmas and Definitions.

\section{Lemmas and Definitions}

In this section, we present several lemmas and definitions which are used in the proof of our main theorem. In this article we prove that every composite numbers, $x^{2}-j$ that $1 \leq j \leq 2 x-2$, between $(x-1)^{2}$ and $x^{2}$ can be written $u^{2}-v^{2}$ or $u^{2}-v^{2}+u-v=(u+1 / 2)^{2}-(v+1 / 2)^{2}=u_{1}^{2}-v_{1}^{2}$ that $u>0$ and $v \geq 0$ and $u-v=u_{1}-v_{1}=q$, where $q$ is a prime number.

Lemma 2.1 All prime factors $q$ where $2 \leq q \leq x$ are Appeared in numbers between $(x-1)^{2}$ and $x^{2}$.

Proof: According to Algorithm division, $x^{2}-j=q s+f$ in which $0 \leq f \leq q-1,1 \leq j \leq x-1$ and $2 \leq q \leq x$, then $1 \leq j+f \leq 2 x-2$.

Lemma 2.2 Every composite number between $(x-1)^{2}$ and $x^{2}$ can be written to $u^{2}-v^{2}$ or $u^{2}-v^{2}+u-v$ where $u>0$ and $v \geq 0$.

Proof: For simplicity we call the composite numbers $x^{2}-j$ as T hereafter. If $\mathrm{T}$ is not prime, then:

$$
T=q f,(q<x)
$$

$\mathrm{q}$ is a prime number and $f$ is a positive integer. Later we show that every composite number between $(x-1)^{2}$ and $x^{2}$ has a prime factor like $q$ so that $q<x$.

$$
f=q+w
$$


If $w$ is an even number so $w=2 v$ otherwise $w=2 v+1$, so:

$$
T=q^{2}+2 q v
$$

Or

$$
T=q^{2}+2 q v+q
$$

Substituting $q$ by $u-v$, we will have

$$
q=u-v
$$

So

$$
T=u^{2}-v^{2}
$$

Or

$$
T=u^{2}-v^{2}+u-v
$$

Definition 1 If $T=u^{2}-v^{2}$ so $T=q(u+v)$, we assume that $u+v=2 x-q+A, A$ is an integer.

Definition 2 If $T=u^{2}-v^{2}+u-v=(u-v)(u+v+1)=u_{1}^{2}-v_{1}^{2}$, in which, $q=(u+1 / 2)-(v+1 / 2)=u_{1}-v_{1}$ so $T=q\left(u_{1}+v_{1}\right)$, we assume that $u_{1}+v_{1}=2 x-q+A, A$ is an integer, $u_{1}=u+1 / 2$ and $v_{1}=v+1 / 2$.

Lemma 2.3 If $(x-m)^{2}-j=q\left(u^{\prime}+v^{\prime}\right)=q\left(2(x-m)-q+A^{\prime}\right)$ and $(x-m-1)^{2}-j_{2}=p$, where $p$ is prime, $A^{\prime}$ is an integer and $m>1$ should be defined earlier in the paper.

Notice: According to the hypothesis of induction there is at least a prime between all squares less than $(x-1)^{2}$.

If $q=1$, then

$$
-j_{1}+j_{2}-A^{\prime}+p=0,1 \leq j_{1} \leq 2 x-2 m-2 \text { and } 1 \leq j_{2} \leq 2 x-2 m-4
$$

Proof: we consider two equation as

$$
(x-m)^{2}-j=q\left(u^{\prime}+v^{\prime}\right)=q\left(2(x-m)-q+A^{\prime}\right) \text { and }(x-m-1)^{2}-j_{2}=p
$$

So we have

$$
(2 x-2 m-1)-j+j_{2}=q\left(2 x-2 m-q+A^{\prime}\right)-p
$$

Then

$$
2 x(q-1)-\left(q^{2}-1\right)+A^{\prime}(q-1)-2 m(q-1)=-j+j_{2}-A^{\prime}+p
$$

For $(x-m)^{2}-j$ to be the prime number in a specific $j=j_{1}, 1 \leq j_{1} \leq 2 x-2 m-2, q$ should be equal to 1 .

So

$$
-j_{1}+j_{2}-A^{\prime}+p=0
$$

Lemma 2.4 Assume that $x^{2}-j^{\prime}=q\left(2 x-q-A_{1}\right)$ and $(x-m-1)^{2}-j_{2}=p$, in which $p$ is prime, and also $1 \leq j^{\prime} \leq 2 x-2$, $A_{1}$ is an integer, then:

$$
2(m+1) x-(m+1)^{2}-j^{\prime}+j_{2}=2 x q-q^{2}+A_{1} q-p
$$

Lemma 2.5 If $l$ to be the number of $2 \leq q<x$ are in $x^{2}-j^{\prime}=$ tq that $1 \leq j^{\prime} \leq 2 x-2$ so $l<x-1$ or $l<\frac{x-1}{q}$, for all $3 \leq q<x$.

Proof: If $q=2$, we put $j^{\prime}=i+2 l(i \geq l)$, so $i+2 l \leq 2 x-2$, then $l<x-1$, but if $q \geq 3$, we put $j^{\prime}=i+2 q l(i \geq l)$, so $i+2 q l \leq 2 x-2$, then $l<\frac{x-1}{q}$, in this case $l$ is the number of $q \geq 3$ that $x^{2}-j^{\prime}=t q$ is odd.

Lemma 2.6 If $f$ to be the number of $p>x$ are in $x^{2}-j^{\prime}=t_{1} p$ that these numbers are odd and $1 \leq j^{\prime} \leq 2 x-2$.

So

$$
f \leq \frac{2 x-2}{9} \cdot \frac{1}{2}
$$

Or

$$
f \leq \frac{x-1}{3} \cdot \frac{1-1 / 9}{5}
$$

Or

$$
f \leq \frac{x-1}{3} \cdot \frac{1-1 / 9-1 / 15}{7}
$$


we continue this method to reach $1-1 / 9-1 / 15-\ldots=0$.

Proof: If $p>x$ and $x^{2}-j^{\prime}=t_{1} p$ to be odd, since $1 \leq j^{\prime} \leq 2 x-2$, so $(x-1)^{2} / q \leq p \leq x^{2} / q$, in which $3 \leq q<x$. Since the distance of between two primes should be at least 2 , so $(x-1)^{2} / q-2 \leq p-2=p^{\prime} \leq x^{2} / q-2, p^{\prime}$ is prime number, but $(x-1)^{2} / q-4 \leq p-4=w \leq x^{2} / q-4, w$ is not prime.

If $q=3$, the number of such $p$ is:

$$
f \leq \frac{2 x-2}{6} \cdot \frac{2}{3} \cdot \frac{1}{2}
$$

but since $p>x$, only one $p>x$ could be in $x^{2}-j^{\prime}=t_{1} p$, so for $q=5, f \leq \frac{x-1}{3} \cdot \frac{1-1 / 9}{5}$. For $q=7, f \leq \frac{x-1}{3} \cdot \frac{1-1 / 9-1 / 15}{7}$, we continue this method to reach, $1-1 / 9-1 / 15-\ldots=0$.

\section{The Proof of Main Theorem}

Theorem There is at least a prime between $(x-1)^{2}$ and $x^{2}$.

Proof: Let we have at least a prime between all squares less than $(x-1)^{2}$. By induction, we prove that, we have a prime between $(x-1)^{2}$ and $x^{2}$. Assume that this is not true, so we can write $x^{2}-j^{\prime}=l q$, i.e all numbers in interval $(x-1)^{2}$ and $x^{2}$ are not primes. Since $1 \leq j^{\prime} \leq 2 x-2$ so according to (Hardy \& Wright, 1964) there is a prime factor like $q$ that for any composite number in interval $(x-1)^{2}$ and $x^{2} \cdot q \leq \sqrt{x^{2}-1}<x$.

Note: If a number in interval $(x-1)^{2}$ and $x^{2}$ like $T$ is not prime so $T$ has a prime factor like $q$ that $q \leq \sqrt{x^{2}-1}<x$. In this section we assume that $j^{\prime \prime}=-j_{2}+h$ that $0 \leq h<q$ and $1 \leq j^{\prime \prime} \leq 2 x-2$, notice that for each number $x^{2}-j^{\prime}$, there is a corresponding divisor $q$. Now we start to prove main theorem: concluding from lemma $2.3,-j_{1}+j_{2}-A^{\prime}+p=0$, we can rewrite below equations:

$$
\begin{gathered}
-A^{\prime}+p-2(m+1) x+(m+1)^{2}=-b+q_{j} t_{j}+j_{1}+j_{2} \\
-A^{\prime}+p-2(m+1) x+(m+1)^{2}=-(b+1)+q_{v} t_{v}+j_{1}+j_{2} \\
\cdot \\
\cdot \\
\cdot \\
-A^{\prime}+p-2(m+1) x+(m+1)^{2}=0+q_{i} t_{1}+j_{1}+j_{2} \\
-A^{\prime}+p-2(m+1) x+(m+1)^{2}=1+q_{f} t_{2}+j_{1}+j_{2} \\
-A^{\prime}+p-2(m+1) x+(m+1)^{2}=2+q_{s} t_{3}+j_{1}+j_{2} \\
\cdot \\
\cdot \\
\cdot A^{\prime}+p-2(m+1) x+(m+1)^{2}=a+q_{u} t_{a}+j_{1}+j_{2}
\end{gathered}
$$

$a, b$ will be determined later.

By substituting the above equations into $-j_{1}+j_{2}-A^{\prime}+p=0$, we have:

$$
\begin{gathered}
-j_{2}+b-\left(j_{2}+2(m+1) x-(m+1)^{2}\right) \equiv 0 \quad\left(\bmod q_{j}\right) \\
-j_{2}+(b+1)-\left(j_{2}+2(m+1) x-(m+1)^{2}\right) \equiv 0 \quad\left(\bmod q_{v}\right) \\
\cdot \\
\cdot \\
\cdot \\
-j_{2}-a-\left(j_{2}+2(m+1) x-(m+1)^{2}\right) \equiv 0 \quad\left(\bmod q_{u}\right)
\end{gathered}
$$

We assume that $j^{\prime \prime}=-j_{2}+h$, in which $-b \leq h \leq a$.

So

$$
j^{\prime \prime}-\left(j_{2}+2(m+1) x-(m+1)^{2}\right) \equiv 0 \quad(\bmod q) \text { that } 2 \leq q<x
$$


According to lemma 2.4, if we substitute the above results into equation:

$$
2(m+1) x-(m+1)^{2}-j^{\prime}+j_{2}=2 x q-q^{2}+A_{1} q-p
$$

Then

$$
j^{\prime \prime}-j^{\prime} \equiv 2 x q-q^{2}+A_{1} q-p \quad(\bmod q) \text { that } 2 \leq q<x
$$

But we should have $j^{\prime} \neq j^{\prime \prime}+q t$, otherwise $q \mid p$, and this is a contradiction unless $q=1$, but we determine $m$ such that $p>x$, so $q \neq p$.

We mention again that $j^{\prime} \not \equiv j^{\prime \prime}(\bmod q)$ and $j^{\prime}=1,2, \ldots, 2 x-2$ since $j_{2}$ is a number between $(x-m-2)^{2}$ and $(x-m-1)^{2}$, then, $1 \leq j_{2} \leq 2 x-2 m-4$.

If $x$ is even, we put $m=\frac{x}{2}$;

If $x$ is odd, we put $m=\frac{x+1}{2}$.

So in $j^{\prime \prime}=-j_{2}+h$, that $-b \leq h \leq a$, we determine $a, b$ such that $j^{\prime \prime}$ is all numbers between 1 to $2 x-2$, or $j^{\prime \prime}=1,2, \ldots, 2 x-2$. But from $j^{\prime \prime}-j^{\prime} \equiv 2 x q-q^{2}+A_{1} q-p(\bmod q)$, we have $x^{2}-j^{\prime \prime} \equiv p(\bmod q)$, that $j^{\prime}=1,2, \ldots, 2 x-2$ and $j^{\prime} \not \equiv j^{\prime \prime}$ $(\bmod q)$, since $x^{2}-p$ is between two squares and according to induction, there is a positive integer like $k$, such that, $x^{2}-p-k=p_{2} \equiv o\left(\bmod q_{u}\right)$, so $q_{u} \mid p_{2}$, Pei's Prime and this is a contradiction unless $q=1$. If for a $j^{\prime \prime},\left|q_{l} t_{s}\right|$ is a prime, for example $\left|q_{l} t_{s}\right|=p_{1}$, so for a $j^{\prime \prime}, x^{2}-j^{\prime \prime}=p+p_{1}$, then for all $j^{\prime \prime}$, that $1 \leq j^{\prime \prime} \leq 2 x-2, x^{2}-j^{\prime \prime}=p+p_{1}$ or $x^{2}-j^{\prime \prime}=p+q t$.

Now we use the above results to reach a contradiction, we use odd statements so:

$$
\left(x^{2}-1 \text { or } 2\right) \ldots\left(x^{2}-(2 x-3) \operatorname{or}(2 x-2)\right)=\left(p+3 t_{1}\right)\left(p+5 t_{2}\right) \ldots\left(p+q t_{1}\right)\left(p+p_{1}\right)\left(p+p_{1}^{\prime}\right) \ldots
$$

but $(x-m-1)^{2}-j_{2}=p$, we assume that $m=x / 2$ so $1 \leq j_{2} \leq x-4$, if $x$ is even and $m=(x+1) / 2$ so $1 \leq j_{2} \leq x-5$, if $x$ is odd.

Hence $p>(x-3)^{2} / 4$, but $x^{2}-j^{\prime \prime}=p+q t>4 p$ and also $x^{2}-j^{\prime \prime}=p+p_{1}>4 p$.

According to lemmas 2.5 and 2.6, we have:

$$
\left(x^{2}-1 \operatorname{or} 2\right) \ldots\left(x^{2}-(2 x-3) \operatorname{or}(2 x-2)\right)<3^{\frac{x-1}{3}} \times \ldots \times q_{a}^{\frac{x-1}{q a}} \times{\frac{x^{2}}{3}}^{\frac{x-1}{9}} \times{\frac{x^{2}}{5}}^{\frac{(x-1)(1-1 / 9)}{3 \times 5}} \times \ldots
$$

We continue to reach $1-1 / 9-1 / 15-\ldots-1 / q_{a}=0$ that $q_{a}<w \ll x, w$ is a positive large integer and $q_{a}$ is a prime number. Hence we have:

$$
\begin{gathered}
(x-1) \log (4 p)<\log \left(x^{2}-1 \operatorname{or} 2\right)+\ldots+\log \left(x^{2}-(2 x-3) \operatorname{or}(2 x-2)\right)< \\
(x-1) \sum_{3 \leq q<w} \frac{\log q}{q}+(x-1)(1 / 9+(1-1 / 9) / 3 \times 5+(1-1 / 9-1 / 15) / 3 \times 7+\ldots+0) \log x^{2}
\end{gathered}
$$

So by refer to (Hardy \& Wright, 1964), $\sum_{3 \leq q<w} \frac{\log q}{q}<\log w+c$, that $c$ is positive constant number, so:

$$
(x-1) \log (4 p)<(x-1) \log w+(x-1) c+0.8(x-1) \log x^{2}
$$

Then for a large $x,(x-1) \log (4 p)<1.7(x-1) \log x$ or $p<x^{1.7} / 4$ and this is a contradiction, because $p>(x-3)^{2} / 4$.

For example, assume that $x=10$, then $m=5$, so $(10-5)^{2}-2=23$ and $(10-5-1)^{2}-3=13$, so $j_{1}=2, p=13, j_{2}=3$, since $-j_{1}+j_{2}-A^{\prime}+p=0$, then $A^{\prime}=14$. But $-j_{2}+h-\left(j_{2}+2(m+1) x-(m+1)^{2}\right) \equiv 0\left(\bmod q_{j}\right)$, since $j^{\prime \prime}=-j_{2}+h$, then $j^{\prime \prime}=-3+h$, so, if we put $h=4,5, \ldots, 21$, we have all numbers between $10^{2}$ and $9^{2}$.

\section{References}

G. H. Hardy, \& E. M. Wright. (1964). An introduction to the theory of numbers. Oxford .

M. EI Bachraoui. (2006). Prime in the interval [2n,3n]. International Journal of Contemporary Mathematical Sciences, 1(3), 617-621.

P. Erdos, \& J. Suranyi. (2003). Topics in the theory of numbers. undergraduate texts in mathematics. Springer Verlag.

P. Erdos. (1932). Beweis eines satzes von tschebyschef. Acta Litt. Univ. Sci., Szeged, Sect. Math., 5, $194-198$. 
Richard K. Guy. (2004). Unsolved problem in number theory. Springer.

Shiva Kintali. (2008). A Generalization of Erdos's Proof of Bertrand-Chebyshev Theorem. http://www.cs.princeton.edu/ kintali.

S. Ramanujan. (1919). A proof of Bertrand, S. Postulate. Journal of the Indian Mathematical Society, 11, 181-182. 